\title{
Abruzzen - im Land des südlichsten Gletschers Europas
}

\author{
Hilke Steinecke \& Peter Schubert
}

\begin{abstract}
Abruzzo is a region in the heart of the Apennines. It is mainly characterized by high mountains. The Abruzzo National Park, Majella National Park and Gran Sasso e Monti della Laga National Park are situated in Abruzzo. The landscape with the massif of Gran Sasso and the immense pastures of Campo Imperatore, their plants and animals are described.
\end{abstract}

\section{Zusammenfassung}

Die Abruzzen sind eine Region im Herzen der Apenninenhalbinsel mit überwiegend Hochgebirgscharakter. Es gibt dort drei Nationalparks, von denen vor allem der Gran Sasso beschrieben wird. Die Landschaft dort mit der Hochebene Campo Imperatore und dem höchsten Berg Corno Grande sowie die Pflanzen- und Tierwelt werden vorgestellt.

\section{Abruzzen, eine landschaftliche Perle Europas}

Die italienische Region Abruzzen (ital. Abruzzo) befindet sich auf der Adriaseite (also im Osten) der Apenninenhalbinsel etwa in Höhe von Rom. Durch die langjährige Verbindung mit dem Königreich Sizilien werden die Abruzzen trotz ihrer relativ nördlichen Lage zu Süditalien gezählt. Landschaftlich haben sie eine große Vielfalt zu bieten, die von den Küsten der Adria mit langen, flachen Sandstränden über das küstennahe Hügelland bis in ein fast $3000 \mathrm{~m}$ aufragendes Hochgebirge reicht. Der größte Flächenanteil der Abruzzen hat Hochgebirgscharakter. Für Italiener sind die Abruzzen ein beliebtes Urlaubsgebiet: im Sommer zum Baden oder Bergwandern und im Winter zum Skifahren. Ausländische Urlauber sind deutlich in der Minderheit. Glücklicherweise ist dieses einzigartige und landschaftlich äußerst attraktive Gebiet bisher vom Massentourismus weitgehend verschont geblieben. Außerhalb Italiens wird dieses Naturparadies als Urlaubziel nur wenig beachtet und ist noch fast ein Geheimtipp. Etwas bekannter geworden sind die Abruzzen vermutlich eher durch das verheerende Erdbeben von L’Aquila im April 2009.

Abb. 1: Im Nationalpark Majella; die Buchen in den unteren Lagen sind bereits grün, darüber in braun die noch kahlen Buchen; auf der dunkelgrünen Fläche wachsen Latschen-Kiefern.

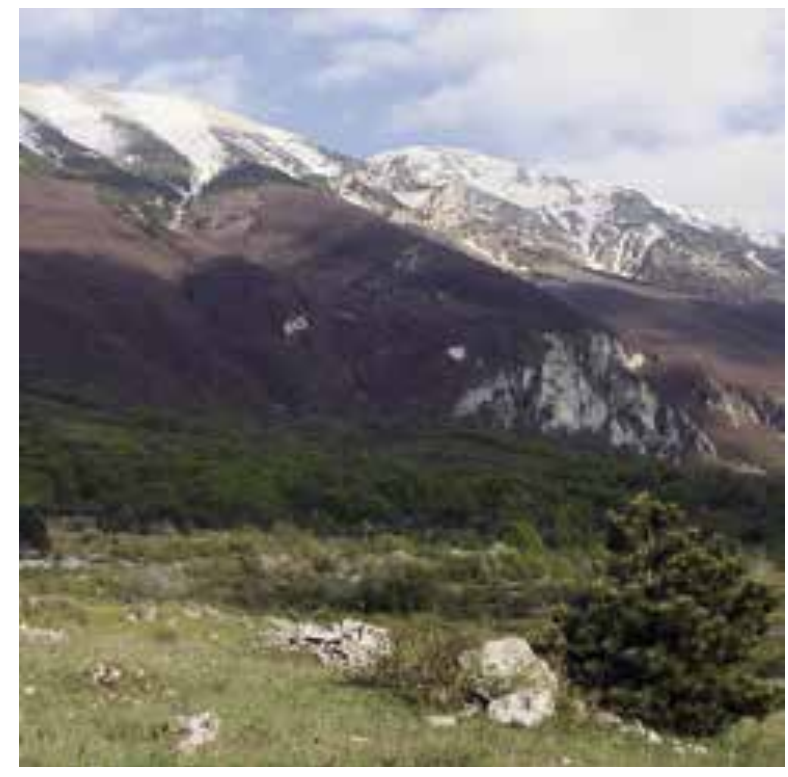

\section{Nationalparks in den Abruzzen}

Für den Natur- und Wanderfreund sind die Abruzzen mit ihrer atemberaubenden Schönheit und ihrer großen Artenvielfalt ein äußerst lohnenswertes Ziel. Von Deutschland kommend ist das Gebiet gut von Westen her über Rom oder von Osten über den Badeort Pescara erreichbar. Im Gebiet gibt es drei große Nationalparks. Der älteste und südlichste ist der Nationalpark Abruzzen. Der Nationalpark Majella umfasst das Majella-Massiv, dessen höchster Berg Amaro 2795 m Höhe erreicht. Hier sind Braunbär, Apennin-Wolf und Steinadler anzutreffen. Der Nationalpark Majella genießt einen ganz besonderen Status, denn er wurde als PAN (Protected-Area-Network)-Park zerti- 


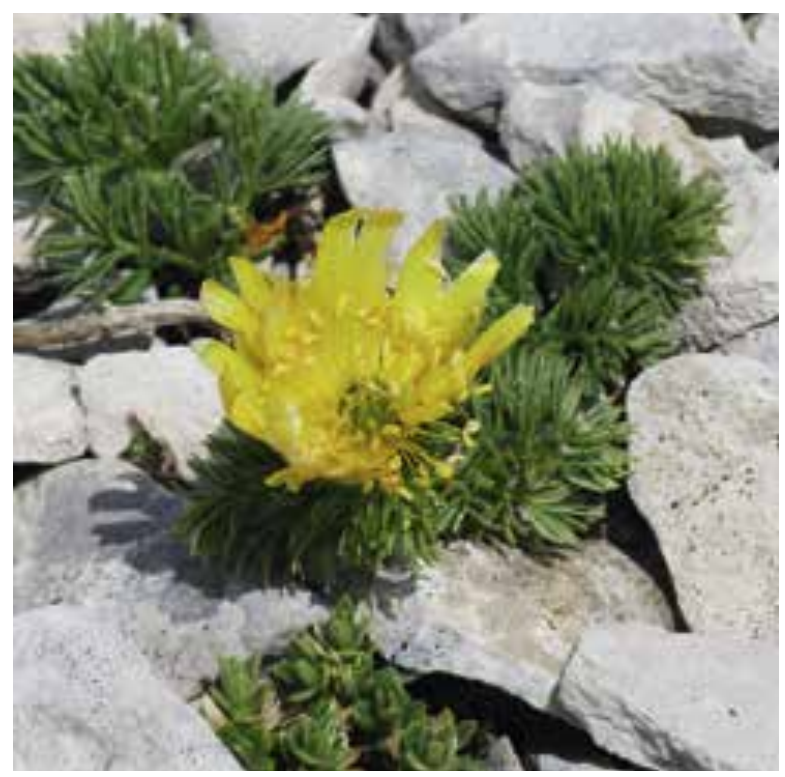

fiziert. Durch Kombination von Naturschutz und nachhaltigem Tourismus sollen hier großflächige europäische „Wildnisgebiete“ dauerhaft geschützt werden. Aktuell wurden bisher 13 Gebiete als PAN-Parks ausgewiesen, die meisten davon befinden sich in Osteuropa. Im Nationalpark Gran Sasso liegt der markante Berg Corno Grande. Mit 2912 m Höhe ist er annähernd so hoch wie Deutschlands höchster Berg, die Zugspitze, und gleichzeitig der höchste Berg Italiens zwischen Alpen und Ätna.

Aufgrund der großen Höhenunterschiede und des ausgeprägten Landschaftsreliefs gibt es in den Bergen der Abruzzen ähnlich wie in den Alpen eine deutliche Vegetationszonierung und dementsprechend eine reiche Flora. Hinzu kommt, dass sich hier biogeographische Regionen wie Alpen, Apenninen und Balkan überlappen. Viele der in den Abruzzen vorkommenden Arten bzw. Unterarten sind Relikte der Eiszeiten, als in dem kalten Klima das Areal vieler Sippen von den Alpen bis nach Südeuropa reichte. Nach den Eiszeiten wurden die Populationen der Hochgebirgspflanzen in den Abruzzen isoliert und es gab keinen genetischen Austausch mehr mit den Populationen der Alpen. Aufgrund dieser Trennung konnten sich im zentralen Apennin neue Arten bzw. Unterarten entwickeln. Für den Nationalpark Majella sind gut 2100 Pflanzenarten bekannt, das entspricht etwa der Zahl von Deutschland. 142

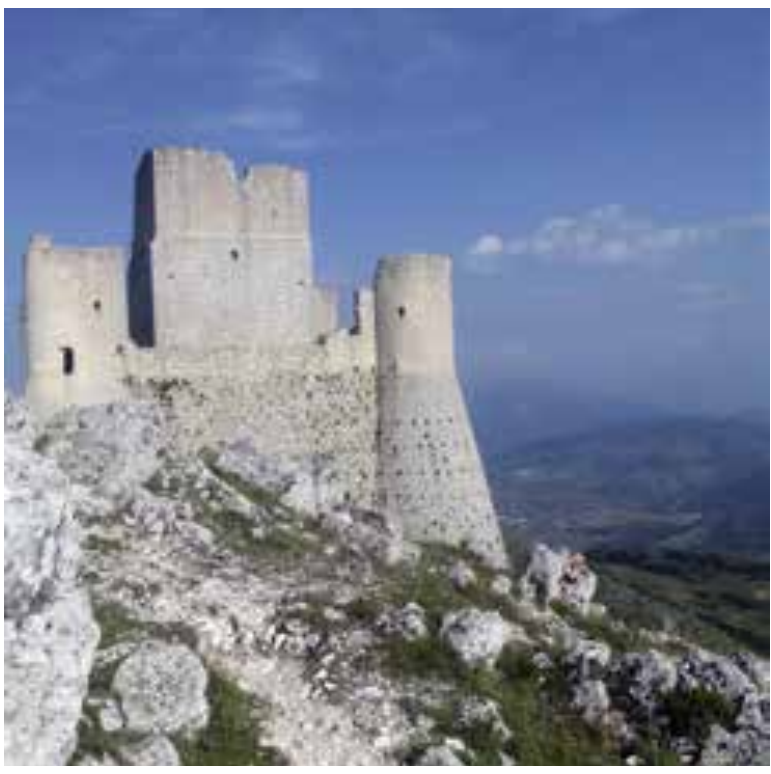

Arten davon sind Endemiten. Einige von ihnen sind in den Abruzzen sehr häufig, kommen aber nur im zentralen Apennin vor. Andere dagegen sind sehr selten wie das auf die MajellaGruppe beschränkte Kleine Alpenglöckchen (Soldanella minima subsp. somnitica; exakte systematische Zuordnung muss noch geklärt werden) oder ein nur zwischen 2400 und $2700 \mathrm{~m}$ Höhe wachsendes winziges Adonisröschen (Adonis distorta).

Um den Rahmen dieses Beitrages nicht zu sprengen, soll nachfolgend genauer nur auf den Nationalpark Gran Sasso eingegangen werden.

\section{Gran Sasso, der "große Stein“}

Der Nationalpark Gran Sasso und Monti della Laga (kurz Gran Sasso, übersetzt „Großer Stein") hat Hochgebirgscharakter, wurde 1991 eingerichtet und umfasst eine Fläche von rund $1500 \mathrm{~km}^{2}$. Er ist eines der artenreichsten Gebiete Europas überhaupt. Wie auch im Nationalpark Majella gibt es besonders an den Nordflanken der Berge beeindruckend ausgedehnte Buchenwälder, die bis rund 1800 m Höhe rei-

\section{Abb. 2 (links): Adonis distorta.}

Abb. 3 (rechts): Majestätisch thront die mittelalterliche Burg Rocca Calascio über dem Tal.

Abb. 4 (Seite 39): Blick über die Ebene Campo Imperatore mit Schmelzwasserflüssen. 
chen und in denen der Apeninnen-Wolf sein Zuhause hat. Für die Buchenwälder steht mit einer durchschnittlichen Jahresniederschlagsmenge von $1300 \mathrm{~mm}$ in den subalpinen Regionen ausreichend Feuchtigkeit für ein üppiges Wachstum zur Verfügung. In der Buchenwaldzone fühlt sich der mitteleuropäische Botaniker fast wie zu Hause, denn kurz vor dem Laubaustrieb blühen hier Geophyten, die auch in Mitteleuropa heimisch sind wie Leberblümchen (Hepatica nobilis), Waldmeister (Galium odoratum), Primel (Primula elatior) und Blaustern (Scilla siberica). Daneben gibt es aber auch Arten, die ihren mitteleuropäischen Verwandten ähneln, entsprechende Lebensräume besiedeln, aber eben nur südlich der Alpen vorkommen. Dazu gehören die Neunblättrige (Dentaria enneaphyllos) und die Vielblättrige Zahnwurz (Cardamine kitaibelii) sowie Apenninen-Anemone (Anemone apennina). Sie sind auch bei uns winterhart und attraktive Frühblüher für den Garten. Aufgrund des Wasserreichtums ist hier nicht nur die Vegetation so üppig und grün; er spielt auch eine wichtige Rolle in der Versorgung der umliegenden Städte mit Trinkwasser.

Die drei Bergketten des Parks sind Gran Sasso d'Italia, Laga und die Monti Gemelli. 57 Gemeinden liegen im Gebiet, die z.T. mittelalterlichen Ursprung haben. Imposant thront über den Bergen in etwa 1500 m Höhe die Burgruine Rocca Calascio, deren Ursprünge auf das 10. Jh. zurückgehen (Touring Club Italiano 2003). Die ehemalige Verteidigungsburg zierte 1980 auch eine 50-Lire-Briefmarke. Idealer Ausgangspunkt für botanische Wanderungen ist der auf $1250 \mathrm{~m}$ Höhe gelegene kleine mittelalterliche Bergort Santo Stefano di Sessanio. Bis in heutige Zeit sind Stadtmauer und MediciPalast Zeugnisse der einst strategisch wichtigen Lage und des Reichtums des Ortes. Nach dem schweren Erdbeben von L'Aquila allerdings ist ein großer Teil der Häuser baufällig, von einem Stützgerüst umgeben und unbewohnbar. Auch der Turm des Medici-Palastes, einst Wahrzeichen des Ortes, wurde durch das Erdbeben zer-

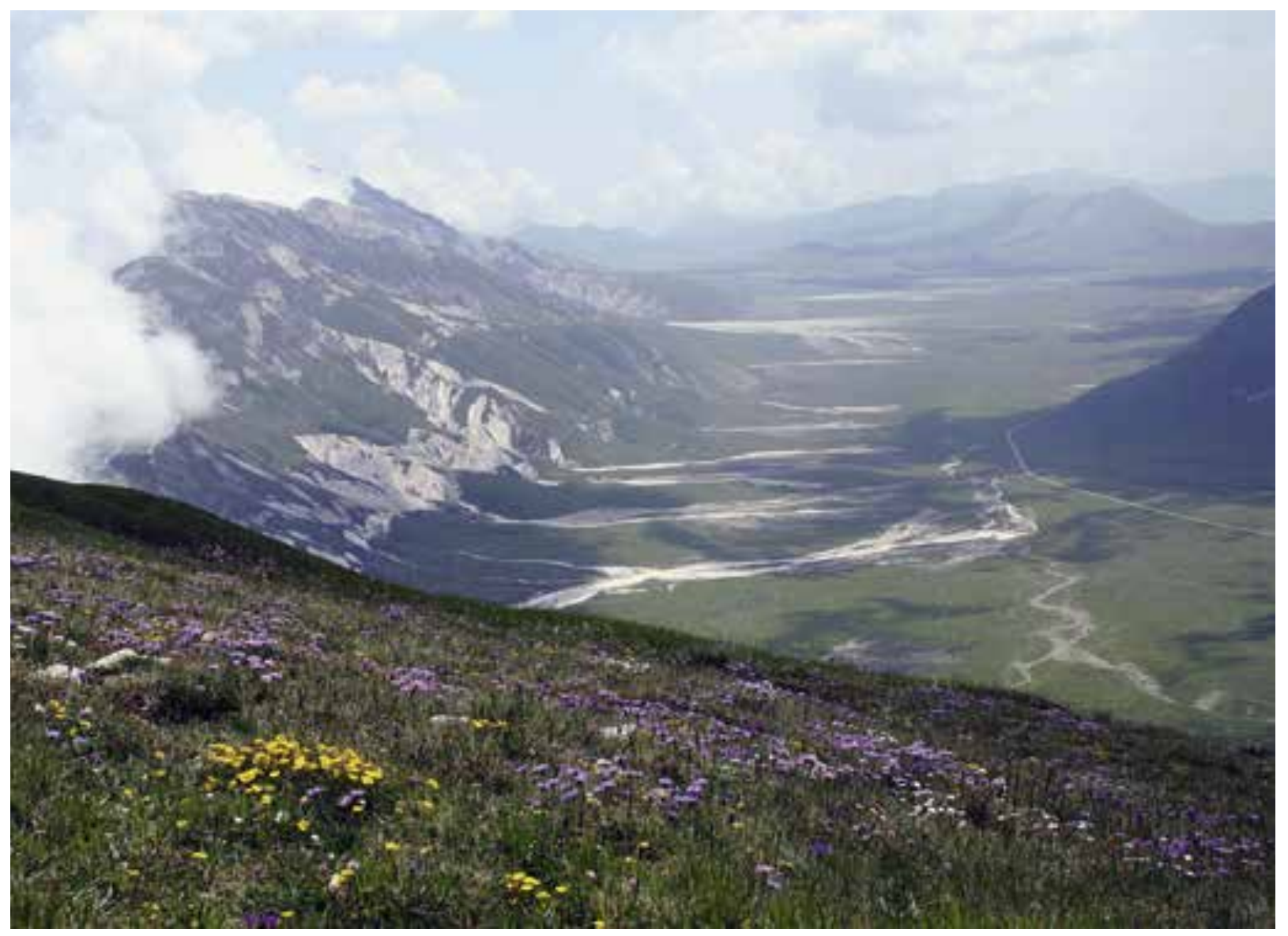




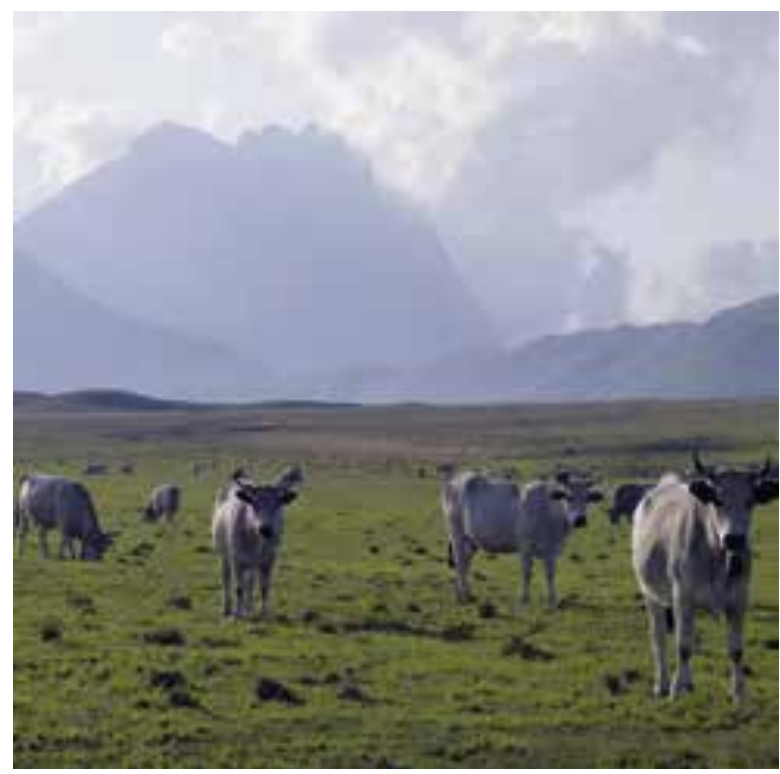

stört und im Jahr 2011 nur noch durch ein Gerüst in Turmform symbolisch angedeutet. Mitglieder der Familie Medici zogen sich in den heißen Sommermonaten in die kühleren Berge zurück. Auf den umliegenden ausgedehnten Trockenrasen ließen sie Schafe halten. Ihre Wolle gilt bis heute als besonders hochwertig; in der Renaissance-Zeit konnten mit der Wolle gute Gewinne erzielt werden. Noch heute erstrecken sich weitläufig um den Ort ausgedehnte artenreiche Trockenrasen, die für Botaniker äußerst lohnenswert sind.

\section{Campo Imperatore, das „kleine Tibet“}

Weiter aufwärts schließt sich eine beeindruckende Hochebene (Campo Imperatore) an. Sie ist die zweitgrößte Hochebene Europas nach der Hardangervidda in Norwegen, ist rund $20 \mathrm{~km}$ lang und etwa $8 \mathrm{~km}$ breit. Benannt wurde das Kaiserfeld, was die wörtliche Übersetzung bedeutet, nach dem Stauferkaiser FRIEDRICH II. (1194-1250), der sich häufig im nahegelegenen L'Aquila aufhielt. Mit dem sich steil darüber wie ein riesiger Kalkklotz erhebenden Corno Grande („Großes Horn“) erinnert das grasbewachsene, z.T. verkarstete und baumlose Hochland an Landschaften im Himalaya, weshalb Italiener es auch als „KleinTibet" bezeichnen. Das Grasland wird als Weideland genutzt; die Hochebene wird intensiv von Rindern und Schafen beweidet, auch frei

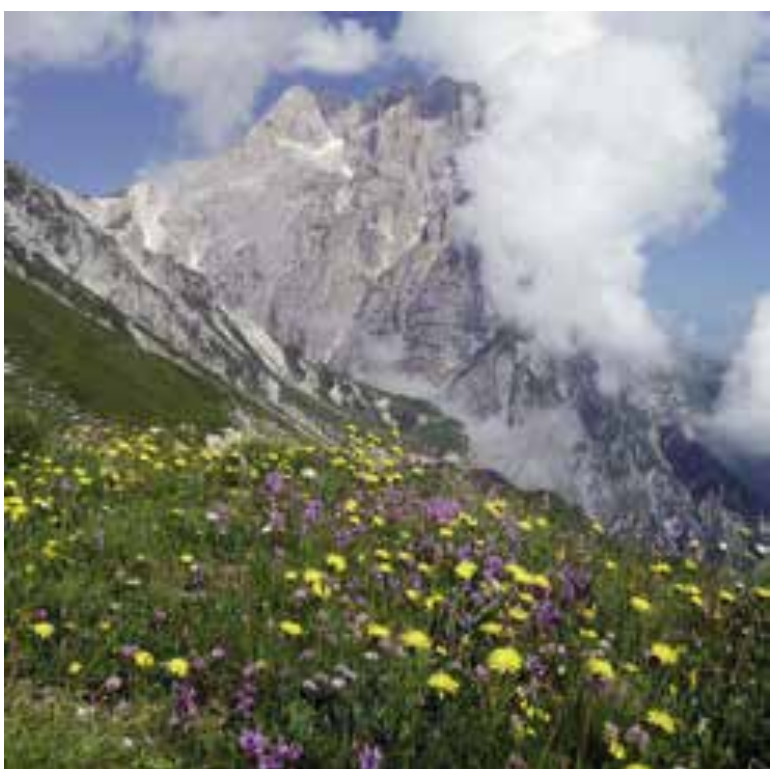

herumstreifende Pferde sind hier anzutreffen. Auf einer Alm kann man frischen Käse erstehen. Der Pecorino-Käse vom Campo Imperatore soll der beste ganz Italiens sein; kein Wunder, denn die Weidetiere können sich hier von vielen verschiedenen aromatischen Wildkräutern ernähren. Die Schäfer kommen heute meist als Saisonarbeiter aus Albanien.

Die beeindruckende Landschaft diente verschiedensten Filmen wie Western und „Im Namen der Rose" als Kulisse. Die Ebene ist so gut wie unbesiedelt, wenige Camping- und Rastplätze laden zum Picknick ein. Am Fuße des Corno Grande befindet sich das Hotel Campo Imperatore, das Ausgangspunkt für viele Wanderungen einschließlich der Besteigung des Corno Grande ist. Seit den 1920er-Jahren ist die Ebene für Italiener auch ein wichtiges Wintersportgebiet. Es gibt dort einzelne Lifte und Skipisten, besonders beliebt ist aber auch das Langlaufen. Der Schnee hält sich bis in das späte Frühjahr hinein. Um den 1. Mai sind die Wiesen auf der Ebene gerade schneefrei, während die Nordlagen der höheren Berge dann meist noch große, dicke Schneefelder aufwei-

Abb. 5 (links): Campo Imperatore mit Corno Grande und Weidevieh.

Abb. 6 (rechts): Bunte Blumenwiesen am Corno Grande. 
sen, sodass dort stellenweise immer noch Ski gefahren werden kann.

Die Hochebene spielte auch in der Geschichte des letzten Jahrhunderts eine Rolle, denn im Hotel Campo Imperatore wurde 1943 der gestürzte Diktator Mussolini für zwei Monate gefangen gehalten. Hier befindet sich zudem eine Außenstelle des Observatoriums von Rom, das seit 1997 mit einem großen Spiegelteleskop ausgestattet ist. In direkter Nachbarschaft gibt es einen kleinen botanischen Garten (gegründet 1952), in dem die wichtigsten Arten der Region kultiviert werden. Der Garten wird von der Universität L'Aquila betreut und ist einer von acht botanischen Gärten in den Abruzzen (Parco Nazionale della Majella 2005).

\section{Wilde Bergwelt}

Die Hochebene wurde in den Eiszeiten durch Gletscher geformt, wovon auch kleinere Schotterhügel, ehemalige Moränen, zeugen. Wie in einem Gletschervorfeld durchziehen noch heute mäandrierende Schmelzwasserflüsse mit ständig wechselndem Bett die Ebene. Diese wird wannenartig von Bergen umschlossen, deren Höhen etwa zwischen 2000 und $2500 \mathrm{~m}$ liegen. Markante Berge, über denen sich der Corno Grande wie ein gewaltiger Kalkklotz erhebt, sind Monte Stella (in manchen Karten auch als Monte Siella bezeichnet, $2027 \mathrm{~m}$ ), Monte Tremoggia (2331 m), Monte Canucia (2564 m), Monte Brancastello (2385 m), Monte Camicia $(2564 \mathrm{~m})$ und Monte Aquila (2494 m). Auf manche Gipfel führen gut markierte Wanderwege, einige sind nur von Kletterern erreichbar.

Auf dem Campo Imperatore und dem Corno Grande herrscht ein Klima mit viel Wind, reichlich Niederschlägen und viel Schnee im Winter, der sich bei Schneeverwehungen durchaus mehrere Meter auftürmen kann, was auch die an den Straßenrändern aufgestellten Schnee-

Abb. 7 (links): Helleborus bocconei.

Abb. 8 (rechts): Gentiana dinarica.
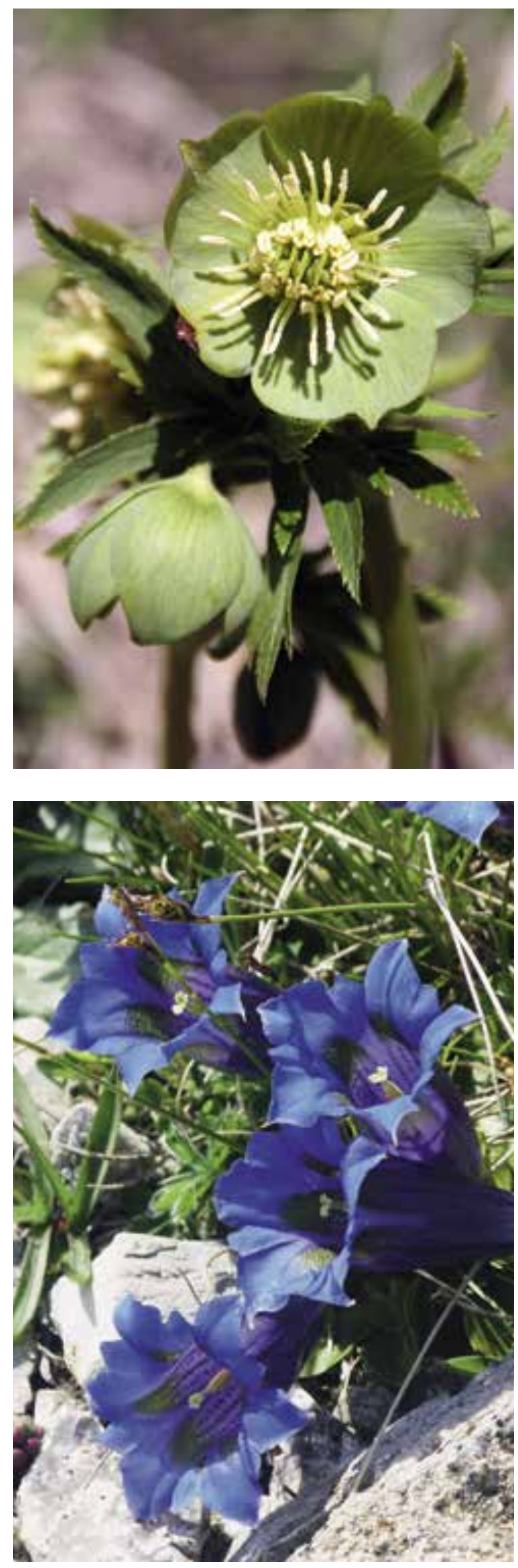


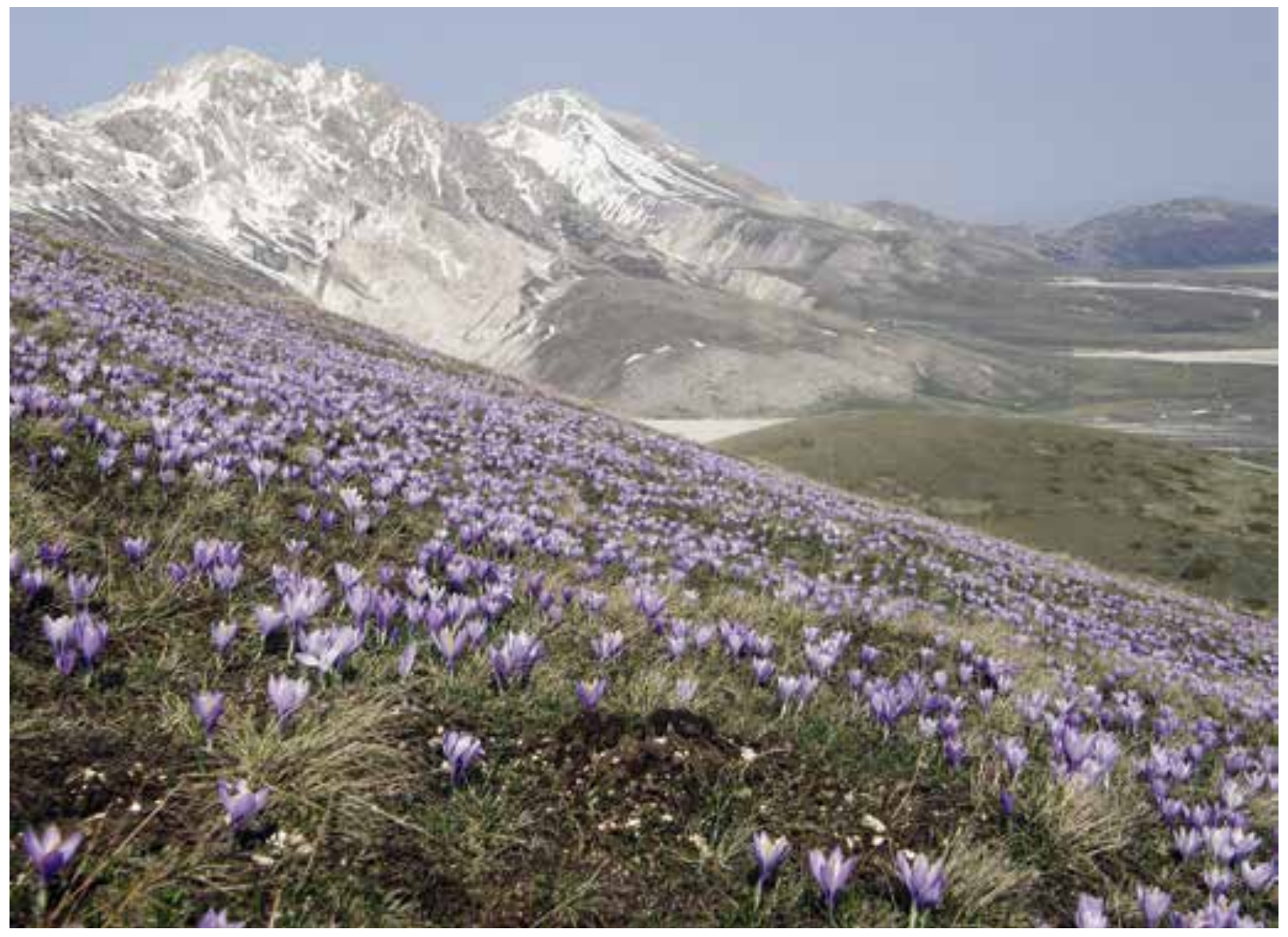

messstäbe erkennen lassen. Die mittlere Jahresniederschlagsmenge an der Klimastation Campo Imperatore (2137 $\mathrm{m}$ ü. NN) beträgt $1143 \mathrm{~mm}$, die Jahresdurchschnittstemperatur beträgt $3,7^{\circ} \mathrm{C}$ und das absolute Minimum beträgt $-27^{\circ} \mathrm{C}$. Kaum ist aber der Schnee verschwunden, erscheinen auf den Wiesen unglaublich üppige Blütenteppiche. Eine der auffälligsten Pflanzen ist Viola eugeniae. Das $5-15 \mathrm{~cm}$ hohe Veilchen wächst dort in Massen und kommt in Höhenlagen zwischen 1000 und $2600 \mathrm{~m}$ vor. Seine Blüten können cremefarben, zitronengelb, violett, hellblau oder mehrfarbig gefärbt sein und erinnern an die der bei uns beliebten Hornveilchen. Es ist mit dem in den Alpen heimischen Gespornten Veilchen (Viola calcarata) eng verwandt und ein Endemit des Zentralapennins. Bei den Einheimischen ist es Tradition, am 1. Mai bei schönem Wetter zum Picknick in die Hochebene aufzubrechen und sich dort üppige Veilchensträuße zu pflücken. Zwischen den Veilchen blühen um diese Jahreszeit auch schon die ersten Polster des
Frühlings-Enzians (Gentiana verna) und die Alpen-Anemone (Anemone alpina). Etwas später escheinen hier zudem die Blüten von Gentiana dinarica, einem Trichter-Enzian, der bei uns als eine beliebte und gut blühende Steingarten-Art kultiviert wird. Er ist auf dem Apennin und dem Balkan in Höhen von 1500-2600 m heimisch. Bevorzugt gedeiht er oberhalb der Baumgrenze, an seiner unteren Verbreitungsgrenze ist er gelegentlich aber auch im Unterwuchs von Buchenwäldern anzutreffen und hat dann nicht so gedrungenen Wuchs wie in den alpinen Lagen.

Abb. 9 (oben): Millionen Krokusse färben nach der Schneeschmelze die Berge rund um die Hochebene violett.

Abb. 10 (Seite 43 oben): Viola eugeniae in verschiedenen Blütenfarben.

Abb. 11 (Seite 43 Mitte): Viola magellensis.

Abb. 12 (Seite 43 unten): Calderone-Gletscher am Nordgipfel des Corno Grande. 
Die botanische Hauptattraktion im Gran Sasso aber ist im Frühling zur Zeit der Schneeschmelze die Massenblüte der Krokusse (meist Crocus vernus). Abermillionen Blüten färben dann die Berge leuchtend violett. Etwa zeitgleich mit den Krokussen erscheinen bis auf $1700 \mathrm{~m}$ Höhe auch die grünen Blüten einer auf dem Apennin endemischen Nieswurz (Helleborus bocconei). Ebenfalls zu den ersten Blühern gehört eine Hundszunge (Cynoglossum magellense), deren dunkel violettrote Blüten von weiß-filzigen Hochblättern umgeben werden, sodass die jungen, noch gedrungenen Blütenstände an große Edelweiß-Köpfchen erinnern. Der Artbeiname magellense, den so manche Art der Abruzzen trägt (z. B. Galium magellense, Viola magellensis), bezieht sich auf das MajellaGebirge (vgl. Tavano 2002).

Im Sommer blühen auf den Wiesen der Ebene dort, wo Schafe und Rinder nicht alles abgefressen haben, mitunter massenweise Orchideen wie z. B. die Pyramiden-Orchis (Anacamptis pyramidalis). Aufgrund des Weidedrucks werden hier besonders solche Arten gefördert, die aufgrund ihrer stechenden Blätter vom Vieh gemieden werden (z.B. die im Gebiet häufige Distel Carduus chrysacanthus) oder wie der Gelbe Enzian (Gentiana lutea) giftig sind.

\section{Alpine Kostbarkeiten in den höchsten Lagen}

Erst ab Juli sind auch auf dem Corno Grande die letzten Schneefelder geschmolzen, sodass der Gipfel bestiegen werden kann. Bergwanderer sollten früh aufbrechen, denn gegen Mittag ziehen häufig Wolken auf, die den Berg umhüllen. Ähnlich wie bei der Passatwolke auf den Kanaren treffen die von der nur $30 \mathrm{~km}$ entfernten Adriaküste aufsteigenden feuchten Luftmassen auf die steilen Berge, sodass es zur Wolken-, Nebel- und Niederschlagsbildung kommt. Nach eigenen Beobachtungen schaffen es die Wolken aber wohl oft nicht, über die Gipfel herüberzusteigen, sodass eine klare Grenze zwischen den dem Meer zugewandten Osthängen und den sonnigen, zur Ebene hin orientierten Bergseiten entsteht. Landschaftlich besonders reizvoll ist der zwar nur noch ziemlich kleine,
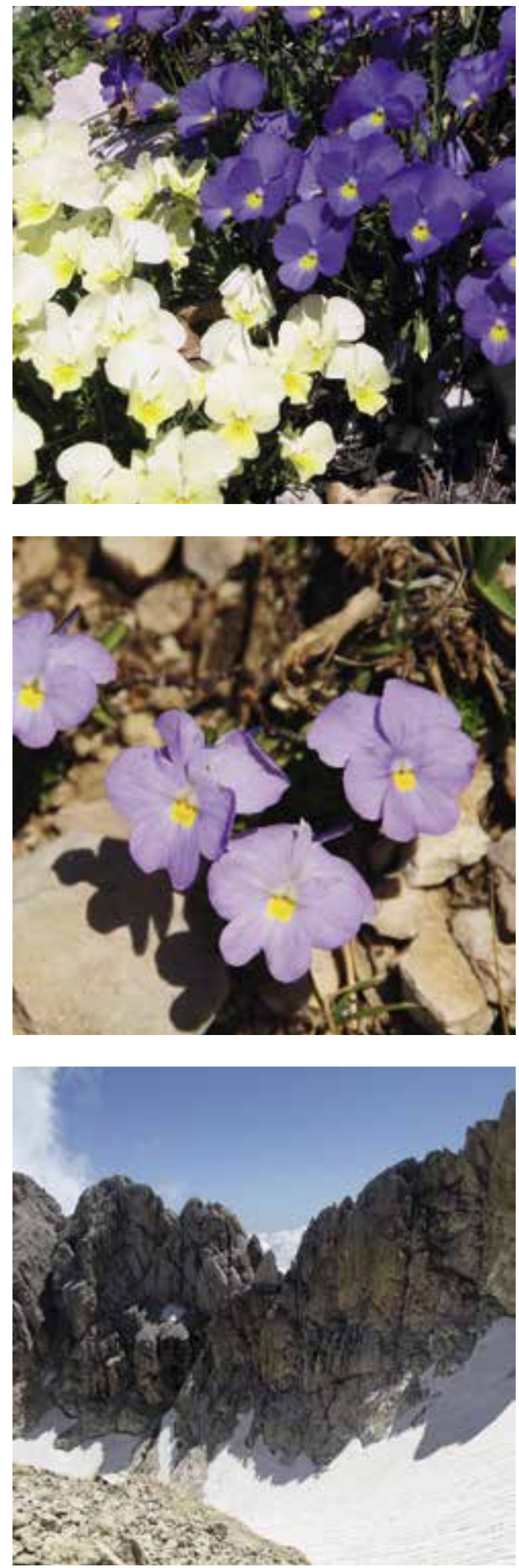

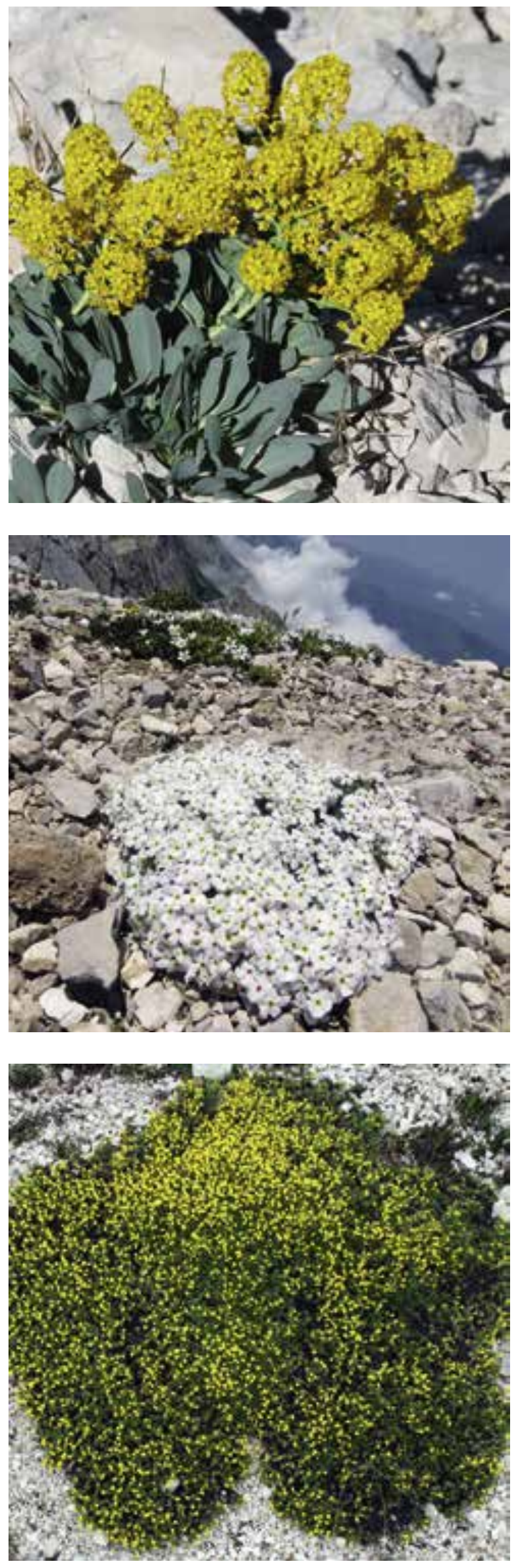

aber immerhin südlichste Gletscher Europas (Calderone-Gletscher) auf der Nordseite unterhalb des Gipfels des Corno Grande.

Im Juli und August liegt hier die Hauptblütezeit der zahlreichen alpinen Pflanzen. In dem Kalk- und Dolomitgeröll der Gipfellagen gedeihen vor allem viele Polsterpflanzen, wie es auch für andere Hochgebirge typisch ist. Die gedrungenen Pflanzen sind im Winter von Schnee bedeckt und dadurch vor Kälte geschützt. Besonders auffällig ist das Apenninen-Mannsschild (Androsace villosa). Die jungen, etwa $0,5 \mathrm{~cm}$ breiten Blüten überziehen das Polster so dicht, dass keine Blätter mehr zu erkennen sind. Die weißen Blüten tragen um den Blütenschlund ein gelbes, ringförmiges Saftmal. Mit zunehmendem Alter verfärben sich die Blüten und auch das Saftmal rosarot. Blütenbesuchende Insekten lernen, Blüten welcher Farbe den meisten Nektar liefern. Gelb blüht dagegen $A n-$ drosace vitalina, ein im zentralen Apennin häufiger Endemit. Die Blüten sind nie richtig weit geöffnet. Kaum mehr als $5 \mathrm{~cm}$ hoch werden die gedrungenen Polster von Myosotis ambigens mit ihren relativ großen, bis $8 \mathrm{~mm}$ breiten himmelblauen Blüten. Die kleinen Blütenkissen erinnern an den seltenen Himmelsherold (Eritrichum nanum) aus den Hochlagen der Zentralalpen. Typische Schuttstauer, die an schotterigen Hängen mitunter dichte Bestände bilden, sind z.B. Steinkraut (Alyssum cuneifolium), Waid (Isatis allionii) und Gamswurz (Doronicum columnae). Der Alpen-Mohn (Papaver alpinum subsp. ernesti-mayeri) ist ein Eiszeitrelikt. Es gibt Populationen mit gelben bzw. weißen Blüten. Immer wieder wird diskutiert, ob es sich bei den Individuen mit unterschiedlicher Blütenfarbe um eine oder zwei verschiedene $\mathrm{Ar}$ -

Abb. 13 (oben): Isatis allionii.

Abb. 14 (Mitte): Androsace villosa.

Abb. 15 (unten): Androsace vitalina.

Abb. 16 (Seite 45 rechts): Leontopodium nivale subsp. nivale.

Abb. 17 (Seite 45 links): Papaver alpinum subsp. ernesti-mayeri. 


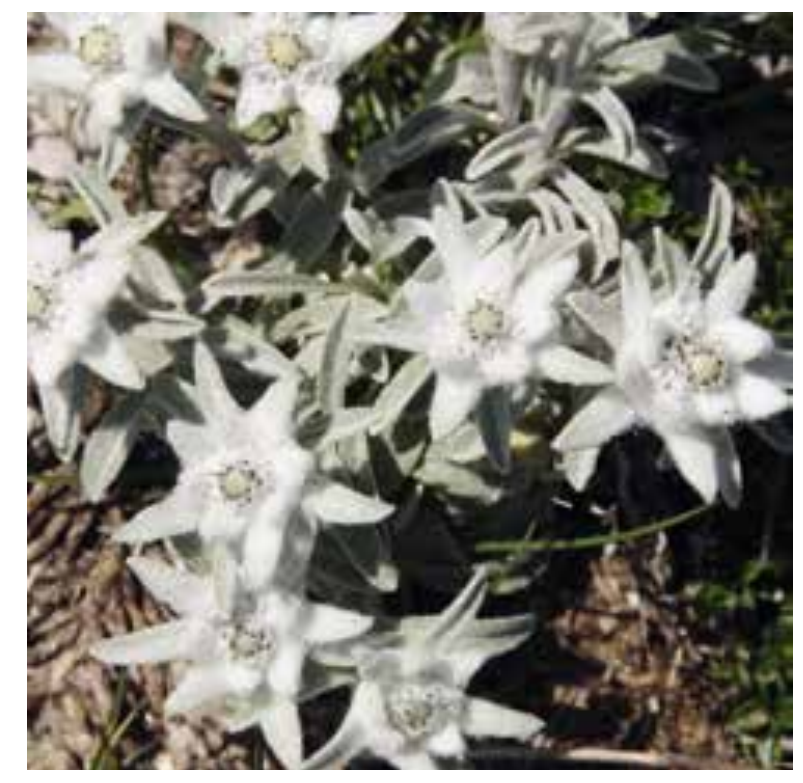

ten handelt. Im Schotter und bis auf $2600 \mathrm{~m}$ Höhe gedeiht auch das Majella-Veilchen (Viola magellensis), ein seltener Endemit, der ein nur sehr kleines Verbreitungsgebiet aufweist (Gran Sasso, Majella, Monte della Laga, Monte Vettore). Im Gegensatz zu Viola eugeniae sind seine Blüten stets blau bis violett gefärbt mit einem zentralen gelben Saftmal. Das untere Kronblatt ist leicht zweilappig ausgerandet.

In Gesteinsritzen oder an steilen Felsen siedeln sich Steinbrech-Arten an. Unverkennbar ist der ebenfalls im Zentral-Apennin endemische Saxifraga porophylla. Den flachen Blattrosetten entspringen nach unten gekrümmte, rot überlaufene und drüsige Blütenstände. An Kalkfelsen bis auf $1800 \mathrm{~m}$ Höhe findet man eine Felsen-Glockenblume (Campanula fragilis). Ihre tassenförmigen Blüten erreichen einen Durchmesser von $3 \mathrm{~cm}$ und sind himmelblau bis weißlich gefärbt. Bei uns lässt sich diese attraktive Glockenblume im Alpinhaus kultivieren (vgl. auch Galetti 2008).

Gut bekannte Arten aus den Alpen sind hier z. B. Silberwurz (Dryas octopetala, ein klassiches arktisch-alpines Florenelement und hier ein Eiszeitrelikt), Gegenblättriger Steinbrech (Saxifraga oppositifolia), Stängelloses Leinkraut (Silene acaulis) und Zwerg-Weide (Salix retusa). Besonders bunt blüht es auf den „Randbergen“ der Ebene, die nur im Gipfelbereich nacktes Geröll aufweisen, ansonsten von niedrigen, blumen-

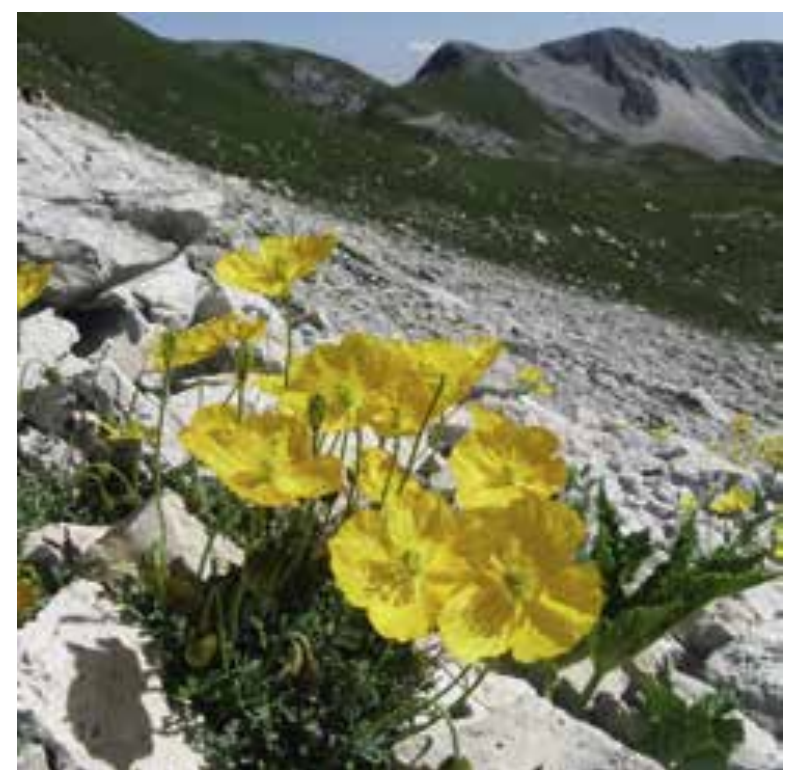

reichen alpinen Matten überzogen sind. Vor allem auf dem Adlerberg (Monte Aquila) sind diese niedrigen Wiesen extrem buntblühend. Hier gedeihen üppig Händelwurz (Gymnadenia conopsea), Großes Kreuzblümchen (Polygala major), Kugelblume (Globularia meridionalis), Alpen-Aster (Aster alpinus var. alpinus) und Büschelglocke (Edraianthus graminifolius). Wo der Boden unter Einfluss von Frostsprengung stark verwittert ist und sich Vegetationslücken gebildet haben, siedeln sich Steinbrech (Saxifraga exarata subsp. ampullacea) oder Hauswurz (Sempervivum riccii) an. Gar nicht selten ist hier das Weiße oder Schnee-Edelweiß (Leontopodium nivale subsp. nivale). Auf dem treffend bezeichneten Monte Stella (ital. Stella alpina $=$ Edelweiß) wachsen sie auf ebener Fläche und deshalb bequem beobachtbar zu Hunderten, wenn nicht sogar zu Tausenden. Je nach Autor werden das Weiße und das Alpen-Edelweiß als zwei verschiedene Arten angesehen oder für Unterarten (nivale und alpinum) von L. nivale eingestuft. Die Verbreitung des Weißen Edelweiß reicht östlich bis nach Monte Negro.

Auch der Tierfreund kommt in den Bergen der Abruzzen auf seine Kosten. Die scheuen und nachtaktiven Wölfe, Bären oder Wildkatzen wird der Wanderer wohl kaum zu Gesicht bekommen, viel wahrscheinlicher aber im schroffen Gelände Pyrenäen-Gämsen (Rupicapra pyrenaica). Ihr Name ist verwirrend, denn 

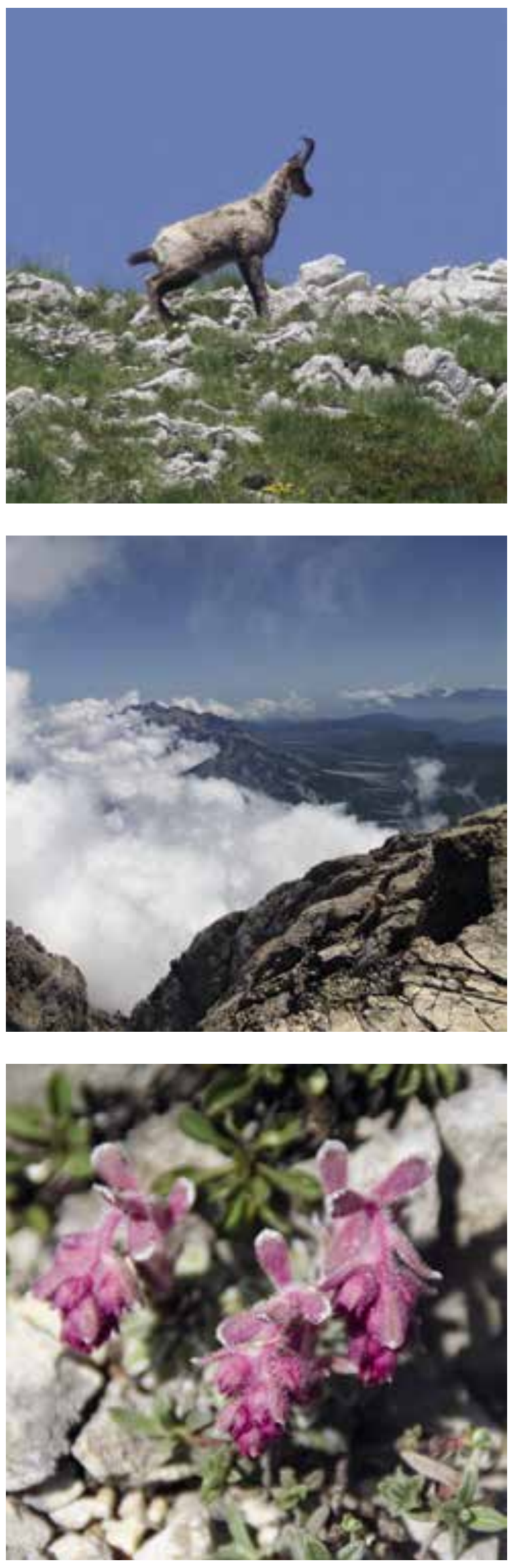

diese Gämsen kommen auch in Nordspanien und im Apennin vor, wo es sich um eine eigene Unterart handelt (Abruzzen-Gämse, Rupicapra pyrenaica ornata). Abruzzen-Gämsen sind selten und im Bestand bedroht. Während des zweiten Weltkrieges hatte sich ihre Zahl auf unter 100 Tiere verringert. Durch besonderen Schutz konnten sich glücklicherweise die Bestände erholen, sodass es heute wieder um die 1000 Gämsen in drei unterschiedlichen Populationen gibt.

Erstaunlich auch, wie viele Schmetterlinge noch in Höhen über $2000 \mathrm{~m}$ anzutreffen sind. Vor allem Kleine Füchse (Aglais urticae) tummeln sich oft zu mehreren auf den Polstern des Stängellosen Leinkrautes. Hoch hinaus kommt auch der Distelfalter (Vanessa cardui), der als Wanderfalter ja auch über die Alpen von Südnach Nordeuropa eingeflogen kommt.

\section{Literatur}

Galetti, G. 2008: Abruzzo in fiore. Ambienti e flora montana della regione dei parchi. - Caramanico Terme. Parco Nazionale della Majella (Hrsg.) 2005: La biodiversità vegetale nelle aree protette in Abruzzo: studit ed esperienze a confronto. - Guardiagrele.

TAvano, G. (Hrsg.) 2002: Guida all‘ alta via della Majella. Natura e presenza umana in un ambiente estremo. Pescara.

Touring Club Italiano (Hrsg.) 2003: Ferien in den Abruzzen. Natur, Kultur, Gastronomie. - Milano.

\section{Karte}

Gran Sasso D’Italia. Carta escursionistica, Edizioni il Lupo, 1:25000.

Abb. 18 (oben): Abruzzen-Gämse.

Abb. 19 (Mitte): Wetterscheide.

Abb. 20 (unten): Saxifraga porophylla. 